\title{
Valor preditivo da dosagem das iodotironinas na avaliação prognóstica de doentes graves
}

\author{
L.S. WARD, *R.M.B. MACIEL
}

Disciplina de Medicina I nterna, Departamento de Clínica Médica, F aculdade de Ciências Médicas da UniversidadeEstadual de Campinas, Campinas, SP; *Disciplina de Endocrinologia, Departamento de Medicina, Universidade Federal de São Paulo - Escola Paulista de Medicina, São Paulo, SP.

RESUMO - Os valores das dosagens de T3 e de T4 diferenciaram os pacientes com boa e má evolução durante a internação em unidade de terapia intensiva.

Овл етіvo. Procurar indicadores para o prognóstico de doentes graves por meio do estudo seqüencial dos níveis séricos dos hormônios tiroidianos.

MÉtodos. Os autores mediram as iodotironinas (T3, T4 e rT3) por ocasião da entrada e da alta de 42 pacientes internados em unidade de terapia intensiva. Verificaram, também, os dados referentes à última coleta de outros 17 doentes, transferidos para a UTI após o início do quadro clínico.

Resultados. Comparando pacientes que evoluíram bem com aqueles que foram a óbito, observaram, nos primeiros, níveis iniciais normais de T4 em $76 \%$ dos casos, valores que se mantiveram está-

\section{INTRODUÇÃO}

Os pacientes portadores de doenças graves, agudas ou crônicas, apresentam uma série de al terações dos níveis dos hormônios tiroidianos. Esses doentes, habitualmente em eutiroidismo clínico, mostram, quase sempre, valores baixos detriiodotironina total (T3), normais de tiroxina total (T4) e elevados de triiodotironina reversa total (rT3) ${ }^{1-9}$. Esta situação, denominada "síndrome do T3 baixo" ou "síndrome do doente grave em eutiroidismo (SDGE)", pode ocorrer em cerca de $70 \%$ dos pacientes internados por doença grave; muitas vezes, este grupo de doentes apresenta não apenas diminuição de T3, mas também de T4 ${ }^{1-9}$. A patogênese destas alterações hormonais ainda não está totalmente esclarecida, sabendo-se que esses doentes possuem uma inibição da 5'-desi odase, enzima que realiza a conversão periférica de T4 em T3, causada por vários fatores, entre os quais se destacam a produção excessiva de corticóides induzida pel o estresse ${ }^{10}$, a di minui ção de captação de T4 pel os tecidos, o que limitaria a disponibilidade de substrato $^{11}$ e a secreção de citocinas ${ }^{12}$. Além disso, a queda do T4 total sérico observada nesses pacientes é devida, provavelmente, à presença de fatores veis ou se elevaram em $65 \%$ dos pacientes durante a internação, de tal forma que níveis normais de T4 estavam presentes em $70 \%$ dos casos por ocasião de sua alta. Ao contrário, $56 \%$ dos pacientes que evoluíram mal já apresentavam T4 inicial baixo, que diminuiu ainda mais em $95 \%$ dos pacientes durante a internação, notando-se valores baixos em $\mathbf{8 1 \%}$ dos casos por ocasião da última amostra. Os valores de T3 e de T4 em conjunto também diferenciaram os pacientes com boa e má evolução.

ConCLUSÃo. Os autores sugerem que a observação dos níveis séricos das iodotironinas pode oferecer importante subsídio na avaliação prognóstica de doentes em estado grave.

UNITERMOS: Hormônios tiroidianos. Síndrome do eutiroidiano doente. Terapia intensiva.

circulantes que competem com o T4 pela ligação à globulina ligadora de tiroxina (TBG), a principal proteína carregadora dos hormônios tiroi dianos ${ }^{13,14}$. O significado fisiopatológico completo dessa diminui ção da conversão periférica de T4 em T3, além da definição do real estado tiroi diano desses pacientes, são questões que ainda permanecem em aberto ${ }^{1}$.

Vários autores têm observado uma estreita relação entre as variações dos níveis dos hormônios tiroidianos com a evolução clínica dos doentes, a ponto de correlacionar os valores hormonais com o seu prognóstico ${ }^{15-18}$. Assim, a queda significativa dos valores de T4 estaria associada a uma evol ução pior, enquanto que os doentes que apresentam T4 estável, geralmente, evoluem bem. Estas observações fornecem um subsídio importante ao intensivista, que poderia, assim, dispor de um parâmetro importante de acompanhamento do paciente em estado grave. Alguns autores têm, também, sugerido a monitorização de doentes crônicos por meio da medida dos hormônios tiroidianos para a indicação de hospitalização, como portadores da síndrome de imunodeficiência adquirida (AIDS) ${ }^{18}$.

Neste trabalho, estudamos as alterações dos hormônios tiroi dianos em pacientes com doenças graves 
internados em unidade de terapia intensiva com o objetivo de estabelecer índices de acompanhamento e/ou prognóstico.

\section{MATERIAL E MÉTODOS}

Estudamos os valores das iodotironinas em 42 pacientes internados em Unidades de Terapia Intensiva (UTI) do Hospital São Paulo da Escola Paulista de Medicina, Universidade Federal de São Paulo. Todos eram portadores de doenças graves que obrigavam o emprego de cuidados intensivos em unidade especializada. Excluímos todos aqueles pacientes portadores de doenças com mal prognóstico a priori, como carcinomatose, traumas craniencefálicos com morte cerebral e intoxicações exógenas irrecuperáveis, assim como aqueles em que havia qualquer evidência ou suspeita de tiroidopatia presente ou pregressa, além dos usuários de drogas que pudessem interferir nos testes de função tiroidiana. Assim, dos 42 doentes estudados, dez encontravam-se em choque de di versas etiol ogias, seis apresentavam processos infecciosos sistêmi cos graves, seis haviam sofrido infarto agudo do mi ocárdio, três eram portadores de insuficiência renal aguda em nível dialítico, nove estavam em coma por trauma craniencefálico, dois apresentavam insuficiência cardíaca congestiva e seis eram politraumatizados graves. Todos os pacientes foram cuidadosamente acompanhados com exames clínicos e dosagens laboratoriais seriadas, desde a sua internação até a alta da UTI. Além desses 42 pacientes, estudados por toda a evolução na UTI, acrescentamos também, neste trabalho, os dados referentes à última col eta de outros 17 doentes, transferidos para a UTI após o início do quadro crítico.

Colhemos diariamente amostras de sangue destes 59 pacientes entre 7 e 9 horas, para dosagem de T3, T4 e rT3 totais, utilizando radioimunoensaios previamente descritos ${ }^{19,20}$.

Realizamos a análise estatística por meio de testes não paramétricos para variáveis não contínuas. Utilizamos o teste do qui-quadrado $\left(\chi^{2}\right)$ para amostras independentes na comparação dos grupos. Fizemos a comparação dos valores iniciais e finais por meio do teste de Wilcoxon em cada grupo de pacientes, com boa ou má evolução. Para analisarmos a evolução dos pacientes para óbito ou alta da UTI, utilizamos o teste de Mann-Whitney.

Consi deramos com boa evol ução os pacientes que receberam alta da UTI e permaneciam em bom estado após 72 horas; com má evolução, consideramos os que morreram durantea internação ou nas 72 horas subseqüentes à alta da UTI.
Tabela 1 - Comparação entre os valores de T4, T3 e rT3 nas amostras iniciais e finais $(x \pm d p)$ entre pacientes que evoluíram para alta da UTI ou para o óbito

\begin{tabular}{|lcccccccc|}
\hline & \multicolumn{2}{c}{$\mathbf{T} 4(\boldsymbol{\mu} \mathbf{g} / \mathbf{d L})$} & & \multicolumn{2}{c}{$\mathbf{T 3}(\mathbf{n g} / \mathbf{d L})$} & & \multicolumn{2}{c|}{$\mathbf{r T 3}(\mathbf{n g} / \mathbf{d L})$} \\
\cline { 2 - 3 } \cline { 7 - 8 } Inicial & $6,3 \pm 2,7$ & $4,6 \pm 2,5$ & & $65 \pm 41$ & $46 \pm 32$ & & $50 \pm 38$ & $82 \pm 47$ \\
Final & $7,0 \pm 2,6$ & $2,4 \pm 1,7$ & & $78 \pm 41$ & $34 \pm 21$ & & $41 \pm 32$ & $64 \pm 30$ \\
\hline
\end{tabular}

Tabela 2 - Comparação entre os valores iniciais e finais do T4 e a evolução dos pacientes para a alta da UTI ou para o óbito

\begin{tabular}{|lccc|}
\hline & $\mathbf{T} 4 \leq 3 \mu \mathbf{g} / \mathbf{d L}$ & $3 \leq \mathbf{T} 4 \leq 5 \mu \mathbf{g} / \mathbf{d L}$ & $\mathbf{T} 4 \geq 5 \mu \mathbf{g} / \mathbf{d L}$ \\
$\mathbf{n}(\%)$ & $\mathbf{n}(\%)$ & $\mathbf{n}(\%)$ \\
Inicial & & & \\
Alta & $3(12)$ & $3(12)$ & $20(76)$ \\
Óbito & $5(31)$ & $4(25)$ & $7(44)$ \\
Final & & & \\
Alta & $1(3)$ & $9(27)$ & $23(70)$ \\
Óbito & $15(58)$ & $6(23)$ & $5(19)$ \\
\hline
\end{tabular}

\section{RESULTADOS}

Notamos, de maneira geral, diminuição do T4 quando o paciente pi orava, e el evação do T4 quando ele apresentava mel hora do quadro clínico. E mbora apresentasse tendência semelhante, o T3 sofreu maiores influências de fatores intercorrentes na doença de base, como picos febris, arritmias, etc. 0 rT3 variou, geralmente, de maneira inversa ao T3, embora seja mais difícil correlacioná-lo com os eventos clínicos. Entretanto, o uso das drogas e os vários fatores intervenientes na própria doença de base ou que a ela se acresceram, como o surgimento de infecções secundárias e a deprivação calórica por deficiente aporte nutricional, além da própria meiavida das iodotironinas dosadas, tornaram extremamente difícil a interpretação dos níveis hormonais no seguimento diário dos doentes. Por este motivo, abstivemo-nos de tais interpretações e utilizamos apenas os dados das amostras iniciais e finais.

Do total de 59 doentes cuj os dados foram analisados, 26 (44\%) evoluíram para óbito, enquanto 33 (66\%) obtiveram al ta da UTI. Os val ores hormonais médios destes dois grupos, tanto no início de sua internação, como por ocasião da última coleta de sangue na UTI, estão representados na tabela 1 , onde observamos que os níveis médios da amostra inicial foram mais el evados nos pacientes com boa evolução do que naqueles que foram a óbito, tanto em relação ao T4 $(p<0,02)$, quanto ao T3 $(p<0,0003)$. Esta diferença continuou quando consideramos as amostras finais de T4 $(p<0,05)$ edeT $3(p<0,002)$. Não observamos diferenças estatísticas em relação ao rT3. 


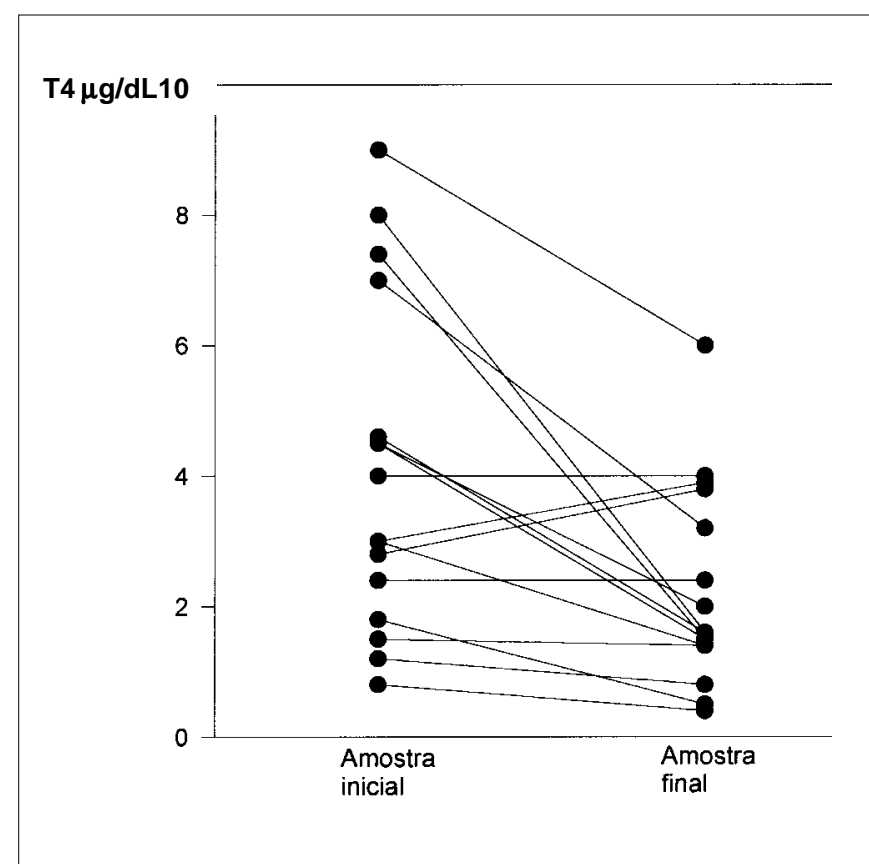

Fig. - Valores de T4 nas amostras inicial e final de 16 pacientes que evoluíram para o óbito. Os valores normais de T4 variam entre 4,5 e $12 \mathrm{mg} / \mathrm{dL}$

Tabela 3 - Comparação entre a tendência dos valores de T4 e a evolução dos pacientes para a alta da UTI ou para o óbito

\begin{tabular}{|lcc|} 
& Manutenção/Incremento & $\begin{array}{c}\text { Decréscimo } \\
\mathbf{n}(\%)\end{array}$ \\
Alta & $17(65)$ & $9(35)$ \\
Óbito & $1(6)$ & $15(94)$ \\
\hline
\end{tabular}

Anal isamos, também, os val ores iniciais efinais de cada hormônio de acordo com a evolução de cada paciente. Assim, os val ores de T4 não apresentaram al ter ações de si gnificância estatística (tabela 2), apesar de se apresentarem inicialmente normais (T4 $\geq$ $5 \mu \mathrm{g} / \mathrm{dL}$ ) em $76 \%$ dos doentes que tiveram boa evolução e diminuídos (T4 entre 3 e $5 \mu \mathrm{g} / \mathrm{dL}$ ) ou muito baixos $(T 4 \leq 3 \mu \mathrm{g} / \mathrm{dL})$ em $58 \%$ dos que foram a óbito. Quando consideramos apenas os val ores da amostra final, no entanto, verificamos que $81 \%$ dos pacientes que evoluíram para o óbito apresentavam valores de T4 abaixo do normal, 2/3 deles em níveis extremamente baixos. Ao contrário, $70 \%$ dos que evoluíram bem possuíam T4 normal ( $p<0,01$ ).

A tendência para uma queda progressiva nos valores de T4 naqueles pacientes que evoluíram mal é bastante marcante, como demonstra a figura. Quando comparamos essa tendência dos valores de T4 com a evol ução dos pacientes (tabela 3), observamos que a queda de T4 prediz 94\% dos óbitos, enquanto que $65 \%$ dos pacientes sobreviventes man-
Tabela 4-Comparação entre os valores iniciais e finais de T3 e a evolução dos pacientes para a alta da UTI ou para o óbito

\begin{tabular}{|lccc|}
\hline & $\begin{array}{c}\text { T3 } \leq 30 \mathbf{n g} / \mathbf{d L} \\
\mathbf{n}(\%)\end{array}$ & $\begin{array}{c}\mathbf{3 0} \leq \mathbf{T} 3 \leq \mathbf{7 0} \mathbf{n g} / \mathbf{d L} \\
\mathbf{n}(\%)\end{array}$ & $\begin{array}{c}\mathbf{T 3} \geq \mathbf{7 0 n} \mathbf{n} / \mathbf{d L} \\
\mathbf{n}(\%)\end{array}$ \\
Inicial & & & \\
Alta & $3(11)$ & $8(31)$ & $15(58)$ \\
Óbito & $5(19)$ & $6(37)$ & $7(44)$ \\
Final & & & \\
Alta & $3(9)$ & $3(9)$ & $27(82)$ \\
Óbito & $5(20)$ & $17(65)$ & $4(15)$ \\
\hline
\end{tabular}

Tabela 5 - Comparação entre os valores iniciais e finais de T3 e T4 e a evolução dos pacientes para a alta da UTI ou para o óbito

\begin{tabular}{lccc}
\hline $\begin{array}{c}\text { T3 } \downarrow \text { e T4 } \\
\mathbf{n}(\%)\end{array}$ & $\begin{array}{c}\text { T3 e T4 nls } \\
\mathbf{n}(\%)\end{array}$ & $\begin{array}{c}\text { T3nIT4 } \downarrow \text { ou T3 } \downarrow \text { T4nl } \\
\mathbf{n}(\%)\end{array}$ \\
$\begin{array}{l}\text { Inicial } \\
\text { Alta }\end{array}$ & $4(15)$ & $14(54)$ & \\
Óbito & $6(37)$ & $4(25)$ & $8(31)$ \\
Final & & & $6(37)$ \\
Alta & $3(9)$ & $20(61)$ & \\
Óbito & $16(62)$ & $3(11)$ & $10(30)$ \\
\hline
\end{tabular}

tiveram ou el evaram seus valores iniciais de T4 $(p<$ $0,05)$. Portanto, a dosagem de T4 por ocasião de internação em UTI e sua observação seqüencial são bons parâmetros prognósticos e evolutivos.

Não observamos relação entre os valores iniciais de T3 e a evolução dos pacientes (tabela 4). Entretanto, ao considerarmos a amostra final, o valor preditivo do T3 normal (T3 $\geq 70 \mathrm{ng} / \mathrm{dL}$ ) é de $82 \%$ para alta da UTI, enquanto que val ores baixos (T3 entre 30 e $70 \mathrm{ng} / \mathrm{dL}$ ) ou muito baixos (T3 $\leq 30 \mathrm{ng} / \mathrm{dL})$ predizem $85 \%$ dos óbitos $(p<0,01)$. Quanto às alterações diárias dos níveis de T3, não observamos relação entre elevação ou queda de T3 com a alta ou óbito.

O rT3 não mostrou qualquer valor prognóstico, quer em relação às amostras iniciais e finais, quer em relação à evolução do paciente.

Final mente, consideramos os dados de T3 e T4 em conjunto, nas amostras iniciais e finais, dividindo os pacientes num primeiro grupo de 23 , que apresentavam níveis hormonais normais de T3 e T4, e num segundo grupo de 19, que tinham valores baixos de T3 e T4 (tabela 5); um terceiro grupo de 17 pacientes não pôde ser caracterizado, por apresentar uma das iodotironinas normal e a outra baixa. Assim sendo, verificamos que oT3 e oT4 estavam normais em $54 \%$ dos que evoluíram bem e em $25 \%$ daqueles que faleceram, não existindo significância destes dados na amostra inicial. Os valores das iodotironinas consideradas em conjunto, entretanto, tornam-se significantes na amostra final $(p<0,01)$. 


\section{DISCUSSÃO}

A literatura vem procurando, há vários anos, índices prognósticos para a determinação do grau de severidade de pacientes portadores de doenças graves; assim, vários parâmetros fisiológicos e dados laboratoriais têm sido utilizados, destacando-se entre el es o APACHE ${ }^{21-26}$. A utilização desses índices é bastante útil, tendo em vista seu emprego na análise da relação custo/benefício referente à internação de determinados doentes em UTI, assim como outros aspectos práticos, como o auxílio ao intensivista na difícil decisão de quais pacientes deveriam ser escoIhidos para a internação nos poucos e altamente requisitados leitos de UTI.

Como mencionado previamente, as alterações na função tiroidiana características da SDGE são freqüentes nos doentes graves, com queda do T3, elevação paralela do rT3 por bl oqueio da conversão periférica do T4 e diminuição do T4, com o eixo hipotálamo-hipofisário mantendo-se íntegro $0^{1-9,27}$. Atribui-se a queda do T3 a um fenômeno adaptativo de proteção do organismo agredido contra a depleção protéica10-12; como já mencionado, a queda do T4 ocorre, também, em virtude da competição, pela TBG, entre o T4 e outras substâncias que atuam concomitantemente no sistema imunológi co durante estados críti cos na evolução de um paciente com uma doença grave ${ }^{13,28-35}$. Outro elemento favorável à teoria que a SDGE é um fenômeno adptativo deriva do trabal ho que demonstra que a reposi ção com T4 não melhora o prognóstico dos pacientes com doença sistêmica grave ${ }^{17}$.

Quanto mais grave o estado do doente, menor o valor do T3 e do T4 circulantes, o que reflete, provavelmente, uma resposta imuno-endocrinológica ampla à doença ou à agressão. Esta relação inversa étão consistente em nossos dados que sugerimos neste estudo, de acordo com outros trabalhos da literatura ${ }^{15-18}$, que os valores finais do T4 e do T3 deveriam ser utilizados como indicadores prognósticos no auxílio às decisões de conduta num paciente grave, como o emprego de suporte nutricional, a necessidade de intervenções invasivas ou até mesmo a alta da UTI. Mais ainda, a tendência apresentada pelo T4 e T3 durante a evolução também poderia ser bastante importante; assim, $64 \%$ dos pacientes que mantiveram os valores de T4 estáveis evoluíram bem, enquanto que morreram $94 \%$ dos doentes cujos valores de T4 decresceram. Os valores de rT3 não demonstraram qualquer valor prognóstico.

O uso de outros indicadores prognósticos de doença grave utilizando dados da função tiroidiana tem sido sugerido recentemente, como o ritmo do TSH empregando metodologia ultra-sensível. O TSH matinal, entretanto, sofre influência de várias drogas, como a dopamina e os gl icocorticói des. Existem, todavia, evidências de que o pico noturno de TSH guarda boa correlação com a evolução dos pacientes $^{36}$.

Em conclusão, com a implantação de métodos de dosagem rápidos nas rotinas hospitalares, acreditamos que a medida das iodotironinas, particularmente do T4, tomada em conjunto com outros sistemas de prognóstico, pode ser de grande utilidade na avaliação de risco de pacientes portadores de doenças graves internados em UTI.

\section{SUMMARY}

\section{Predictive value of the measurement of iodoti- ronines on the prognosis of patients with severe nonthyroidal illnesses}

PuRPose. In order to find prognostic parameters in patients with severe diseases, we analyzed sequentially the levels of thyroid hormones.

METHODS. We measured iodothyronines (T3, T4 and rT3) in 42 patients beforetheadmission and after the discharge in an intensive care unit. In addition, we also measured the iodothyronines in other 17 patients after the discharge.

REsults. Comparing the group of good outcome with the patients who died, we observed in theformer group initial normal T4 levels in $76 \%$ of the patients, which were maintained in $65 \%$ of them during hospitalization and in $70 \%$ of them at the time of delivery from the intensive care unit. Patients who died, however, presented initial low T4 levels in 56\% of them, decreasing values in 95\% of them during hospitalization and low levels in $81 \%$ of patients at the last dosage. The combined profile of T3 and T4 also differentiated good and bad outcome.

ConcLusion. We suggest that serial analysis of serum levels of thyroid hormones may help the evaluation of critical care patients. [Rev Ass Brasil 1997; 43(2): 114-8.]

KEY-WORDS: Thyroid hormones. Euthyroid sick syndrome. Intensive care units.

\section{REFERÊNCIAS BIBLIOGRÁFICAS}

1. Wong TK, Hershman JM. Changes in thyroid function in nonthyroid illness. Trends Endocr Metab 1992; 3: 8-12.

2. Nicoloff J T, Lo Presti J S. Nonthyroidal illness.In Braverman LE, Utiger RD (eds): Werner and Ingbar The Thyroid, 6th ed. Philadel phia, Lippincott, 1991; 357-68.

3. Chopra IJ , Hershman J M, PardridgeWM, N icol off J T. Thyroid function in non-thyroidal illness. Ann Intern Med 1983; 98 : 946-57. 
4. Hershman J M, Pardridge WM, Nicoloff JT, Chopra IJ . Thyroid function in nonthyroidal illnesses. Ann Intern Med 1983; 98: 946-57.

5. Maciel RMB, Vieira J GH, Dib SA, Russo EMK. Síndrome do T3 baixo com eutiroidismo em doença sistêmica severa. Rev Ass Med Brasil 1983; 29: 63-6.

6. Russo EMK, Vieira J GH, Muniz J RS, Sterian Ward L, Maciel RMB. Testes de função tiroidiana em pacientes eutiroidianos com doença sistêmica grave. Arq Bras Endocrinol Metabol 1983; $125-8$.

7. Wartofsky L, Burman K. Alterations in thyroid function in patients with systemic illness: the euthyroid sick syndrome. Endocr Rev 1982; 3: 164-217.

8. Schussler GC. Thyroid function tests in patients with nonthyroidal diseases. Thyroid Today 1980; 3: 1-6.

9. Ingenbleck $Y$. Thyroid function in nonthyroidal illness. In De Visscher M (ed): TheThyroid, 1st ed., New York, Raven Press, 1980; 499-527.

10. Bianco AC, Nunes MT, Hell NS, Maciel RMB. The role of glucocorticoids in the stress-induced reduction of extrathyroidal 3,5,3'-triiodothyronine generation in rats. Endocrinology 1987; 120: 1.033-8.

11. Denardin OVP. Influência do diabetes mellitus experimental na conversão periférica de T4 em T3: estudo com perfusão de fígado em ratos. Tese de Doutorado, Escola Paulista de Medicina, 1986.

12. Pang XP, Hershman J M, Mirell CJ , Pekary AE. I mpairment of hypothalamic-pituitary-thyroid function in rats treated with human recombinant tumor necrosis factor $\alpha$ (cachectin). Endocrinology 1989; 125: 76-84.

13. Chopra If , Solomon DH, Chua Teco GN, Eisenberg J B. An inhibitor of the binding of thyroid hormones to serum proteins is present in extrathyroidal tissues. Science 1982; 215: 407-9.

14. Openheimer J H, Schwartz HL, Mariash CN, Kaiser FE. Evidence for a factor in the sera of patients with nonthyroidal disease which inhibits iodothyroninebinding by solid matrices, serum proteins, and rat hepatocytes. J Clin Endocrinol Metab 1982; 757-66.

15. Slag MF, Morley J E, Elson MK et al. Hypothyroxinemia in critically ill patients as a predictor of high mortality. J AMA 1981; 245: 43-5.

16. Kaptein EM, Weiner J M, Robinson WJ , Wheeler WS, Nicol off J T. Relationship of altered thyroid hormone indices to survival in nonthyroidal illness. Clin Endocrinol 1982; 16: 565-74.

17. Brent GA, Hershman J M. Thyroxine therapy in patients with severe nonthyroidal illness. Low serum thyroxine concentration. J Clin Endocrinol Metab 1986; 63: 1-8.

18. Lo Presti J, Fried J C, Spencer CA, Nicoloff J T. Unique alterations of thyroid hormone indices in the acquired imunodeficiency syndrome (AIDS). Ann Intern Med 1989; 110: $970-5$.
19. Russo EMK, Vieira J GH, Maciel RMB, Fonseca RMG. Desenvolvimento e caracterização de métodos de radioimunoensaio para dosagem de iodotironinas (T4, T3 e rT3). Arq Bras Endocrinol Metabol 1982; 26: 23-8.

20. Russo EMK, Maciel RMB, Vieira J GH. Radioimunoensaio de 3,3',5'-triiodotironina (T3 reverso no soro. Metodol ogia e aplicações clínicas. Rev Bras Patol Clin 1983; 19: 59-66.

21. Cullen DJ, Ferrara LC, Briggs BA, Walker PF, Gilbert J . Survival, hospitalization charges and follow-up results in critically ill patients. N Engl J Med 1976; 294: 982-7.

22. Shoemaker WC, Chang P, Czer L. Cardiorespiratory monitoring in postoperative patients. I. Predictor of outcome and severity of illness. Crit Care Med 1979; 7: 237-44.

23. Walfish PG, Arrejo H, Israel Y, Blake J, Kalant H. Serum triiodothyronine and other clinical and laboratory indices in alcoholic liver disease. Ann Intern Med 1979; 91:13-9.

24. Knaus WA, Draper EA, Wagner DP, Zimmerman J E. APACHE II; a severity of disease classification system. Crit Care Med 1985; 13: 818-29.

25. Chang RWS, J acobs S, Lee B. Use of APACHE II severity of disease classification to identify intensive care unit patients who would not benefit from total parenteral nutrition. Lancet 1986; 1: 1.483-7.

26. Zimmerman J E. APACHE III study design: analytic plan for evaluation of severity and outcome in intensive care unit patients. Crit Care Med 1989; 17: 169-75.

27. Faber J Kukergaard C, Rasmunsen $\mathrm{H}$ et al. Pituitary-thyroid axis in critical illness.J Clin Endocrinol Metab 1987; 65: 315-20.

28. Scovill J E, Saba TM, Kaplan J E, Bernard HR, Powers J r SR. Disturbances in circulating opsonic activity in man after operative and blunt trauma. J Surg Res 1977; 22: 709-16.

29. Constantian MB, Menzoian J O, Nimberg RB, Schmid K, Mannick J A. Association of a circulating immunosuppressive polipeptide with operative and accidental trauma. Ann Surg 1977; 185: 73-9.

30. McLoughlin GA, Wu AU, Saporoschetz I, Nimberg RB, Mannick J A. Correlation between anergy and a circulating immunosuppressive factor following major surgical trauma. Ann Surg 1979; 190: 297-303.

31. Kaplan J E, Scovill WA, Bernard H, Saba TM. Reticuloendothelial phagocytic response to bacterial challenge after traumatic shock. Cir Shock 1977; 4: 1-8.

34. Ozawa M, Sato K, Han CD et al. Effects of tumor necrosis factor alpha-cachectin on thyroid hormone metabolism in mice. Endocrinology 1988; 123: 1.461-7.

35. Gupta S. Study of activated T cells in man. II. Interleukin 2 receptor and transferrin receptor expression on $\mathrm{T}$ cells and production of interleukin 2 in patients with acquired immunodeficiency syndrome (AIDS) and AIDS-related complex. Clin I mmunol I mmunopathol 1986; 38: 93-100.

36. Arem R, Deppe S. Fatal nonthyroidal illness may impair nocturnal thyrotropin levels. Am J Med 1990; 88: 225-32. 